OPEN ACCESS

Edited by:

Ashok Kumar

University of Florida, United States

Reviewed by:

Ekaterina Dobryakova, Kessler Foundation, United States

Lijun Bai,

Xi'an Jiaotong University, China

*Correspondence:

Mingzhou Ding

mding@bme.ufl.edu

Received: 24 July 2018 Accepted: 27 September 2018 Published: 19 October 2018

Citation:

Burke SE, Babu Henry Samuel I, Zhao Q, Cagle J, Cohen RA, Kluger B and Ding M (2018) Task-Based Cognitive Fatigability for Older Adults and Validation of Mental Fatigability

Subscore of Pittsburgh Fatigability

Scale. Front. Aging Neurosci. 10:327.

doi: 10.3389/fnagi.2018.00327

\section{Task-Based Cognitive Fatigability for Older Adults and Validation of Mental Fatigability Subscore of Pittsburgh Fatigability Scale}

\author{
Sarah E. Burke'1, Immanuel Babu Henry Samuel2, Qing Zhao², Jackson Cagle², \\ Ronald A. Cohen ${ }^{3}$, Benzi Kluger ${ }^{4}$ and Mingzhou Ding ${ }^{2 *}$
}

\begin{abstract}
'Department of Neuroscience, College of Medicine, University of Florida, Gainesville, FL, United States, ${ }^{2}$ J. Crayton Pruitt Family Department of Biomedical Engineering, Herbert Wertheim College of Engineering, University of Florida, Gainesville, FL, United States, ${ }^{3}$ Department of Clinical and Health Psychology, College of Public Health and Health Professions, University of Florida, Gainesville, FL, United States, ${ }^{4}$ Departments of Neurology and Psychiatry, Anschutz School of Medicine, University of Colorado, Aurora, CO, United States
\end{abstract}

Cognitive fatigue and cognitive fatigability are distinct constructs. Cognitive fatigue reflects perception of cognitive fatigue outside of the context of activity level and duration and can be reliably assessed via established instruments such as the Fatigue Severity Scale (FSS) and the Modified Fatigue Impact Scale (MFIS). In contrast, cognitive fatigability reflects change in fatigue levels quantified within the context of the level and duration of cognitive activity, and currently there are no reliable measures of cognitive fatigability. A recently published scale, the Pittsburgh Fatigability Scale (PFS), attempts to remedy this problem with a focus on the aged population. While the physical fatigability subscore of PFS has been validated using physical activity derived measures, the mental fatigability subscore of PFS remains to be tested against equivalent measures derived from cognitive activities. To this end, we recruited 35 older, healthy adult participants (mean age $73.77 \pm 5.9$ ) to complete the PFS as well as a prolonged continuous performance of a Stroop task ( $>2$ h). Task-based assessments included time-on-task changes in self-reported fatigue scores (every $20 \mathrm{~min}$ ), reaction time, and pupil diameter. Defining subjective fatigability, behavioral fatigability, and physiologic/autonomic fatigability to be the slope of change over timeon-task in the above three assessed variables, we found that the PFS mental subscore was not correlated with any of the three task-based fatigability measures. Instead, the PFS mental subscore was correlated with trait level fatigue measures FSS ( $\rho=0.63$, $p<0.001)$, and MFIS cognitive subsection ( $\rho=0.36, p=0.03$ ). This finding suggested that the PFS mental fatigability subscore may not be an adequate measure of how fatigued one becomes after a given amount of mental work. Further development efforts are needed to create a self-report scale that reliably captures cognitive fatigability in older adults.

Keywords: cognitive fatigability, Stroop task, fatigue, performance measures, validation 


\section{INTRODUCTION}

Fatigue is a common complaint in older adults and is associated with poor quality of life, functional disability, and increased mortality (Cathébras et al., 1992; Gill et al., 2001; Hardy and Studenski, 2008a,b). Despite the apparent importance of fatigue as a public health concern, research in this area has long been hampered by problems associated with definition and measurement (Muscio, 1921). To address this issue, recent work has suggested a unified taxonomy for more precisely communicating and describing the construct of fatigue (Kluger et al., 2013). Important aspects of this taxonomy include: (1) Distinguishing subjective fatigue (feelings of lack of energy or increased effort) from objective performance fatigability (changes in performance over time); (2) distinguishing fatigue from related phenomena (e.g., depression and sleepiness); (3) specifying what domains of performance are affected by fatigue (e.g., cognitive or physical); and (4) describing what physiologic factors are associated with fatigue.

The concept of fatigability, reflecting the change in selfreported fatigue or behavior as a result of a given activity, is particularly relevant to older adults as highly fatigable individuals may limit their activities in an effort to reduce subjective fatigue (Eldadah, 2010). Given the well-documented adverse consequences of limiting one's activities, there is thus a great need to develop and validate measures of both performance and subjective fatigability, which are essential for diagnosis and treatment.

The Pittsburgh Fatigability Scale (PFS) represents recent efforts to meet this need. Its physical fatigability subscore demonstrated good concurrent and convergent validity against physical performance-based measures of behavioral and perceived physical fatigability for the aging population (Glynn et al., 2015). Its mental fatigability subscore, however, has not been validated against any cognitive performance-based metrics. The goal of the current study was thus to assess the construct validity of the PFS mental fatigability subscore by determining whether it was associated with task-based subjective, behavioral, or physiological fatigabilities. Here, subjective fatigability was measured by the rate of change of serial self-reported fatigue scores during the prolonged performance of a cognitive task; behavioral fatigability was measured by the rate of change in reaction times over time-on-task; physiologic/autonomic fatigability was measured by the rate of change in pupil diameter as a result of participants fatiguing (Hopstaken et al., 2015).

\section{MATERIALS AND METHODS}

\section{Participants}

Thirty-five older adults ( $>60$ years of age) were recruited through newspaper advertisements and flyers. Participants were compensated for their time with a $\$ 50$ gift card for each visit. The Fatigue Severity Scale (FSS) (Krupp et al., 1989) score was collected during the initial phone screening. Participants were eligible for the study if they were free from diagnoses of either neurological disorders or non-neurological disorders that might contribute to fatigability, including the following: cardiac, respiratory, endocrine disorders; currently receiving treatment for cancer; severe depression; attention deficit disorder; or sleep disorders. All participants were native English speakers and were free from any reading, hearing, or vision impairments.

\section{Baseline Assessments}

Participants were tested for subjective, objective, and behavioral dimensions of fatigue and fatigability over the course of two visits. For the first visit, all participants completed the PFS, a 10-item scale in which they were asked to rate the imagined fatigue level, from a score of 0 (no fatigue) to 5 (extreme fatigue), that would arise from participation in activities of specific duration and intensity (Glynn et al., 2015). Mental and physical subscores were summed separately. Participants were also asked to complete other questionnaires (Table 1) including the FSS, a 9-item scale to assess degree of fatigue symptoms, the Modified Fatigue Impact Scale (MFIS) (Fisk et al., 1994), a 21-item scale to assess the effects of fatigue on cognition, physical activity, and psychosocial functioning, the 36-Item Short Form Survey (SF36) (Ware and Sherbourne, 1992) measuring general well-being, the Hospital Anxiety and Depression Scale (HADS) measuring depression symptoms, the Pittsburgh Sleep Quality Index (PSQI) (Buysse et al., 1989) measuring sleep quality, and the Epworth Sleepiness Scale (ESS) (Johns, 1991) measuring sleep quality. Cognitively, participants were given a brief test of cognitive function, the Montreal Cognitive Assessment (MOCA) (Nasreddine et al., 2005), and a computerized test of executive working memory function, the Operation Span task (OSPAN) (Unsworth et al., 2005).

\section{Cognitive Fatigability Task}

During the second visit, participants completed a cued Stroop task for a sustained $160 \mathrm{~min}$ without break (Figure 1). Every 20 min during the task, participants were presented with a fatigue scale and asked to rate the current fatigue level from 1 to 10 . Five participants were excluded from the analysis as they asked to quit within $1 \mathrm{~h}$ of task start. One additional participant was excluded from the reaction time analysis for data recording issues.

TABLE 1 | Measures collected on the first visit (baseline).

\begin{tabular}{ll}
\hline Instruments & Domain assessed \\
\hline FSS (Krupp et al., 1989) & Fatigue \\
Modified fatigue impact scale (MFIS) (Fisk et al., 1994) & Fatigue \\
36-Item short form survey (SF36) (Ware and & General health \\
Sherbourne, 1992) & \\
$\begin{array}{l}\text { Pittsburg sleep quality index (PSQI) (Buysse et al., 1989) } \\
\text { The epworth sleepiness scale ESS (Johns, 1991) }\end{array}$ & Sleep quality \\
$\begin{array}{l}\text { Hospital anxiety and depression scale (HADS) (Zigmond } \\
\text { and Snaith, 1983) }\end{array}$ & Sleepiness \\
$\begin{array}{l}\text { Pittsburgh sleep quality index (PSQI) (Buysse et al., } \\
\text { 1989) }\end{array}$ & Sleep amount \\
$\begin{array}{l}\text { Montreal cognitive assessment (MOCA) (Nasreddine } \\
\text { et al., 2005) }\end{array}$ & Cognition \\
The operation span task (OSPAN) (Unsworth et al., & Working memory
\end{tabular}
2005) 


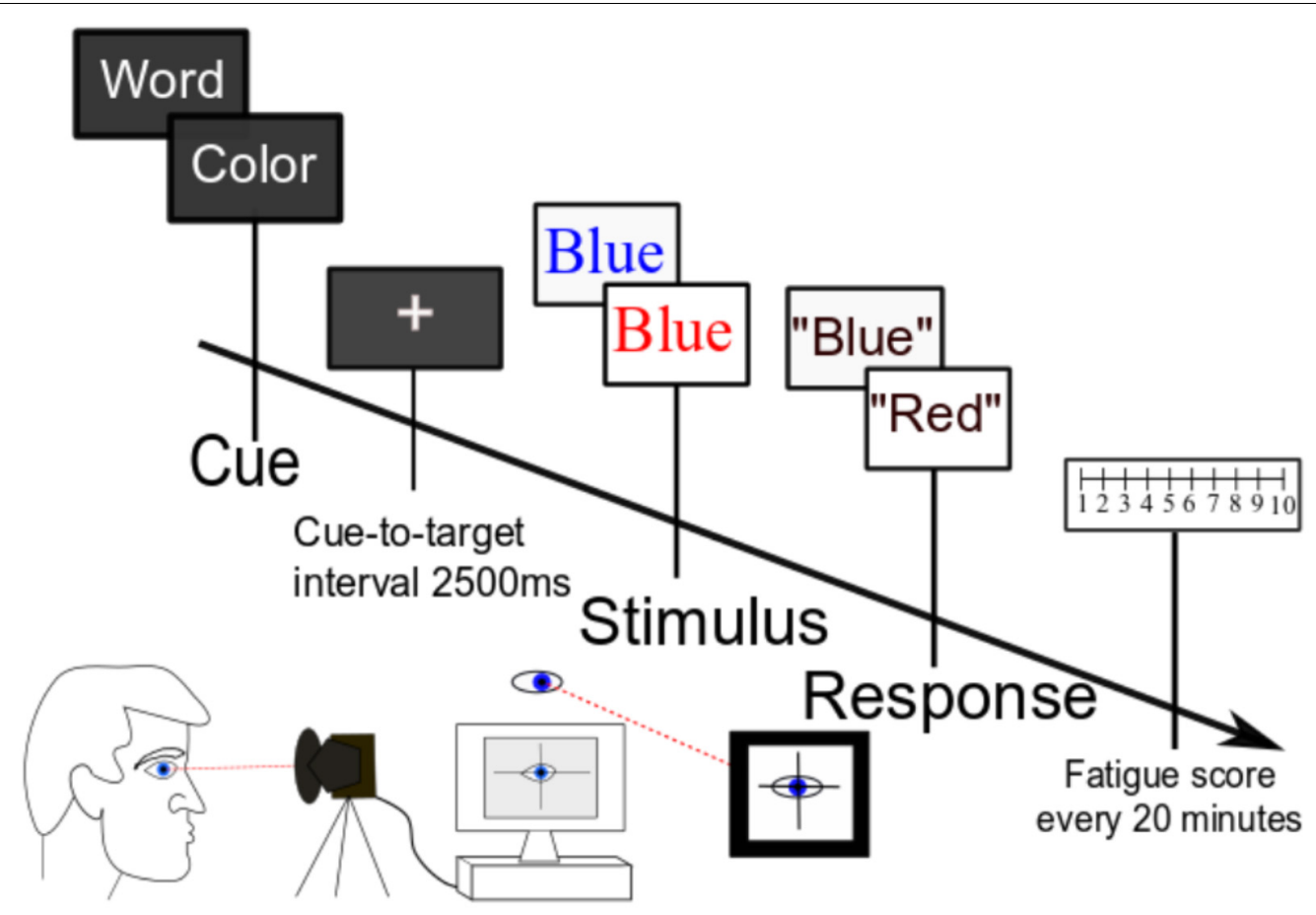

FIGURE 1 | Task paradigm. Participants performed a cognitively demanding cued Stroop task for $2.5 \mathrm{~h}$ without break. Serial subjective fatigue scores, reaction time data, and pupil diameter were recorded continuously throughout the experiment.

As shown in Figure 1, at the beginning of each trial, participants were presented with a cue of either "color" (50\% of trials) or "word" (50\% of trials). Following a 2500 -ms cueto-target interval, they were then presented with a target word in either a congruent font (the color of the font matches the meaning of the written word) or an incongruent font (the font color does not match the meaning of the word). If the trial is cued for "color," the subject's task was to name the color of the font and disregard the meaning of the written word; if the trial is cued for "word," the subject's task was to read the word and ignore the font color. The next trial started when the experimenter recorded the response uttered by the participant. We chose the Stroop task because cognitive fatigue is associated with impairment of executive function (van der Linden et al., 2003), and this task stresses executive control over conflict processing (Jensen and Rohwer, 1966). Further, the Stroop task has been shown to reliably induce fatigue in our prior studies of young participants (Wang et al., 2014).

The Stroop task was programmed in the Presentation software. Pupil diameter was monitored using an Eye Tracker (SR Research EyeLink 1000), which was synced to the Presentation software. Continuous pupil data was sampled at $1000 \mathrm{~Hz}$ and epoched from $-200 \mathrm{~ms}$ to $3500 \mathrm{~ms}$ for each trial. We were mainly interested in changes in tonic pupil diameter rather than stimulus-related changes in pupil diameter. Thus, pupil diameter in the precue time period $(-200 \mathrm{~ms}$ to $0 \mathrm{~ms})$, normalized to the baseline condition at the beginning of the task, was analyzed for time-on-task changes. Four participants were excluded from pupillometry analysis for excessive head movements.
To minimize variability, all participants were coached on the behavioral task by one designated experimenter with a uniform script and tested at 9:00 AM. They were asked to get a usual night's sleep prior to the visit and verified that they were able to do so on the morning of the task. They were also asked to avoid caffeine on the day of testing. Participants were provided with a chin rest to ensure that the head stayed in the same position throughout the task. They were also provided with a pillow for back support to help keep the upper body stationary. Further, they were reminded to keep head movements to a minimum during trials and to keep their gaze and head position in line toward a continuous central fixation point on the screen.

Task-based fatigability was defined in several domains by the rate of change of the pertinent variables. Subjective fatigability was calculated to be the slope of the fatigue scores over the course of the experiment (rate of change). The slope of reaction time change over the experiment duration was calculated as a measure of behavioral fatigability. Physiologic/autonomic fatigability was calculated as the rate of change in pupil diameter.

\section{Statistical Analysis}

Error trials, trials with reaction time less than $500 \mathrm{~ms}$ or longer than $4000 \mathrm{~ms}$ were excluded from analysis. A mixed linear model for repeated measures was applied to fatigue rating, reaction time, and pupilometry data. Survey and scale data were collected via Redcap software package and analyzed in MATLAB. Relations among survey/scale data and behavioral, perceptive, and autonomic fatigability indices were analyzed using Spearman's rank correlation, controlling for the effect of age 


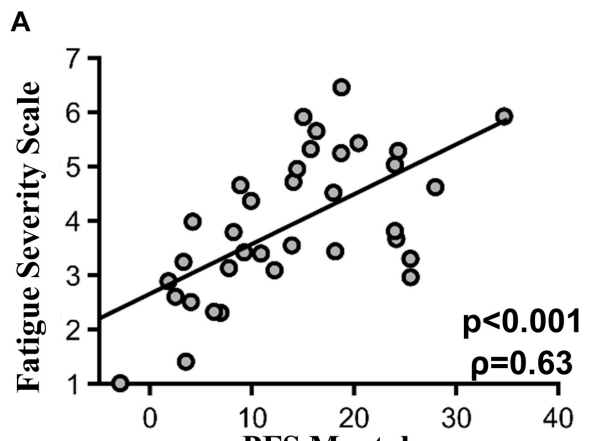

C

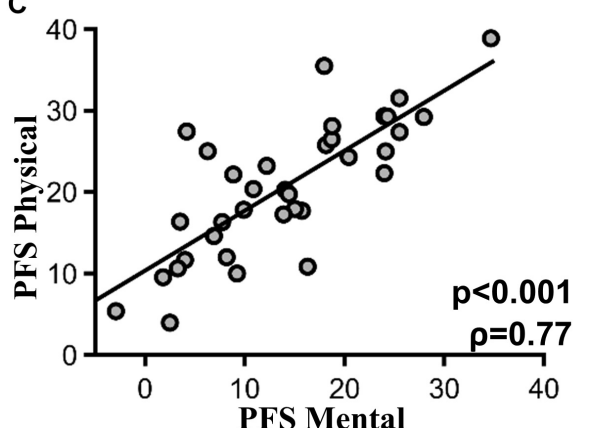

B

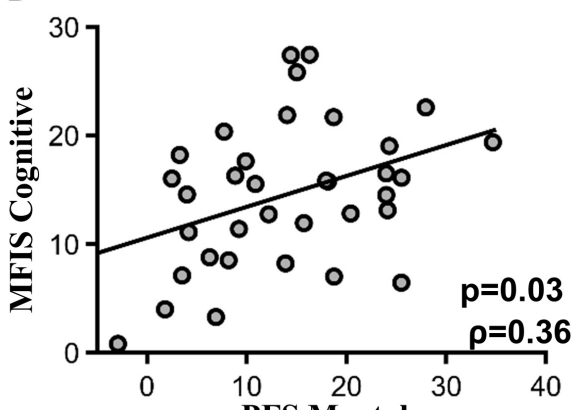

D

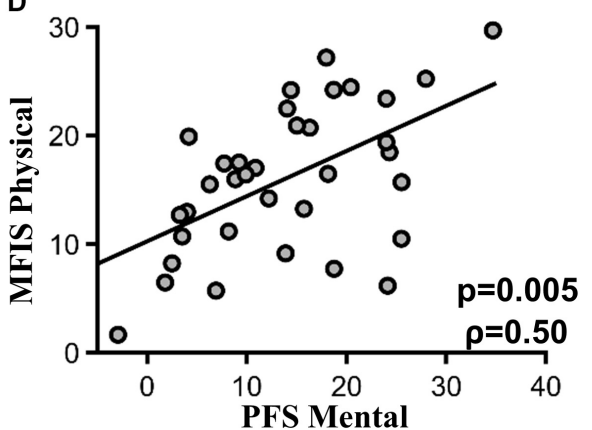

FIGURE 2 | PFS mental subscore and other fatigue scales. (A) Pittsburgh mental fatigability subscores and FSS ( $p<0.001, \rho=0.63$ ). (B) Pittsburgh mental fatigability subscores and MFIS cognitive subscore $(p=0.03, \rho=0.36)$. (C) PFS mental and physical subscore $(p<0.001, \rho=0.77)$. (D) PFS mental subscore and MFIS Physical subscore $(p=0.005, \rho=50)$.

and depression (HADS). Multiple comparisons were corrected with Bonferroni-Holm (Holm, 1979) correction at the alpha value of 0.05 .

\section{RESULTS}

Thirty-five older adults (14 male; $40 \%$ ), mean age $73.77 \pm 5.90$ with a range of 63-84, participated in the study. Trait fatigue levels, based on the FSS (Krupp et al., 1989), ranged from 1.116.44 with mean score of $3.94 \pm 1.53$. PFS mental subscores had a mean of $14.00 \pm 9.82$ and ranged from 0 to 35 . Scores did not differ between gender $(p=0.98)$, with mean score being $14.29 \pm 8.42$ for male and $13.81 \pm 9.36$ for female, and there was no effect of age $(p=0.67)$.

\section{Relation Between PFS Mental Fatigability Subscores and Other Self-Report Measures}

PFS mental fatigability subscores were highly correlated with the FSS scores $(\rho=0.63, p<0.001)$ (Figure 2A). PFS mental fatigability subscores were also correlated with the MFIS cognitive subscore $(\rho=0.36, p=0.03)$ (Figure 2B). In addition, PFS mental fatigability subscores were correlated with PFS physical fatigability subscore $(\rho=0.77, p<0.001)$ (Figure 2C), as well as MFIS physical subsection score $(\rho=0.50, p=0.005)$ (Figure 2D). However, no correlations (or weak correlations) were found with the baseline health surveys such as SF36 general health questionnaire $(\rho=-0.37, p=0.11)$ or depression scale (HADS) $(\rho=0.41, p=0.09)$, or with the sleep surveys such as ESS $(\rho=.41, p=0.09)$ or PSQI $(\rho=0.29, p=0.26)$. There was no correlation between PFS mental fatigability subscore and the MOCA score $(\rho=-0.05, p=0.80)$ and the OSPAN score $(\rho=-0.20, p=0.52)$.

\section{Relation Between PFS Mental Fatigability Subscore and Task-Based Subjective Fatigability}

As time-on-task progressed, subjective fatigue ratings increased significantly, as assessed across 20-min time blocks, where $F_{(8,325)}=5.83, p<0.001$, and effect size $>3$ (Figure 3A). At the individual subject level, linear fit to subjective fatigue scores as a function of time-on-task was calculated, and the slope was used as a measure of subjective fatigability (Figure 3B). PFS mental fatigability subscore and subjective fatigability were not significantly correlated $(\rho=-0.07, p=0.71)$ (Figure 3C).

\section{Relation Between PFS Mental \\ Fatigability Subscore and Task-Based Behavioral Fatigability}

As time-on-task progressed, RT slowed from the first 20-min time block to the last, where $F_{(7,169)}=3.71, p=0.001$, and effect size $=0.24$ (Figure 3D). Accuracy did not decline significantly, where $F_{(7,160)}=0.691, p=0.68$, and effect size $=0.39$. At 

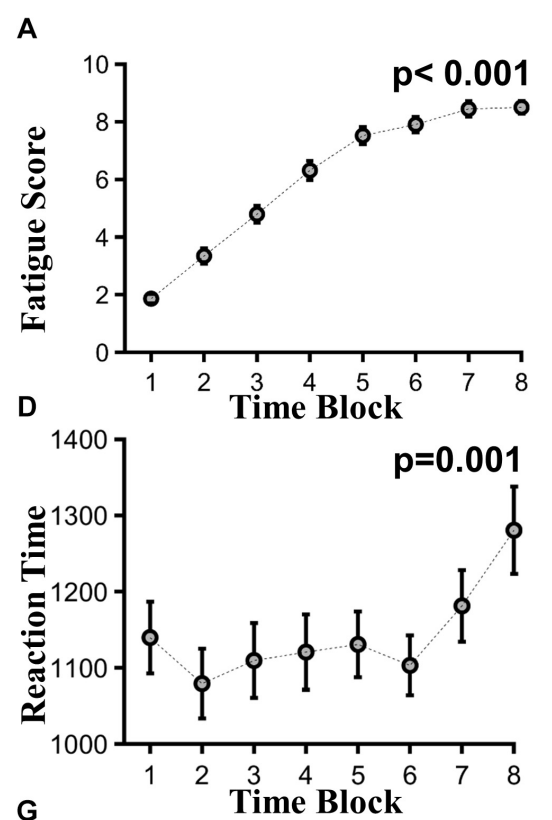

G

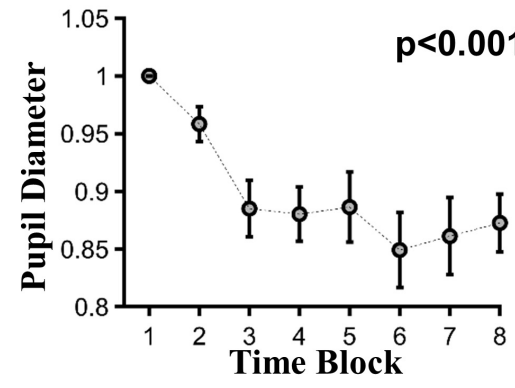

B

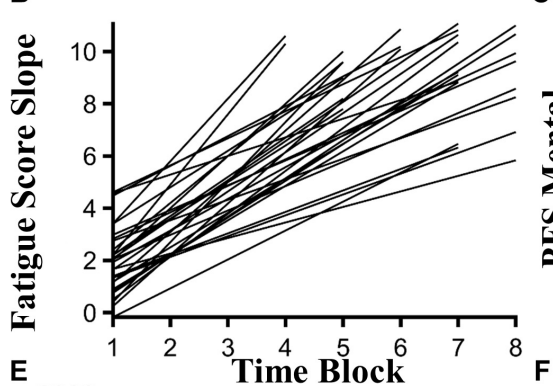

C

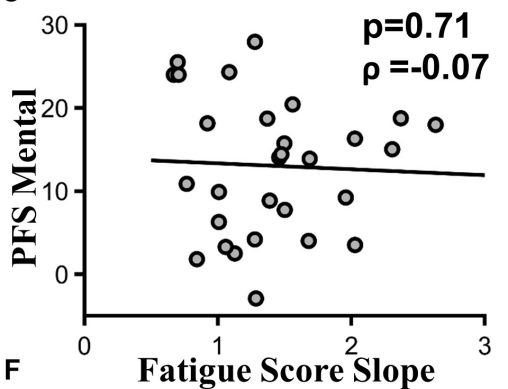

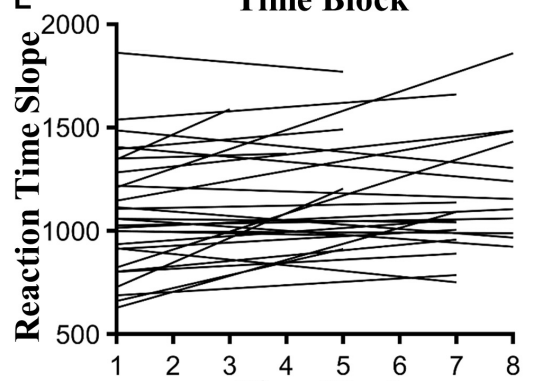

H

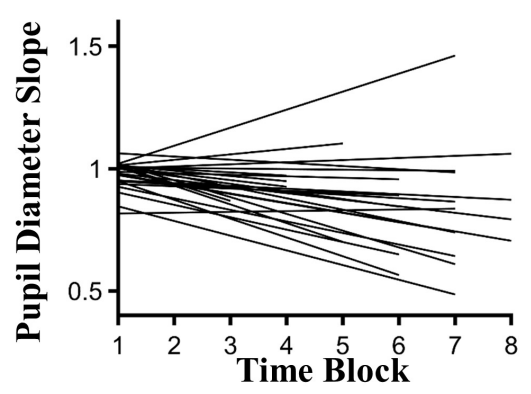

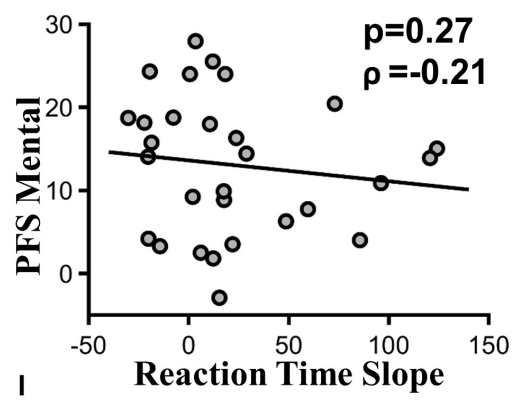

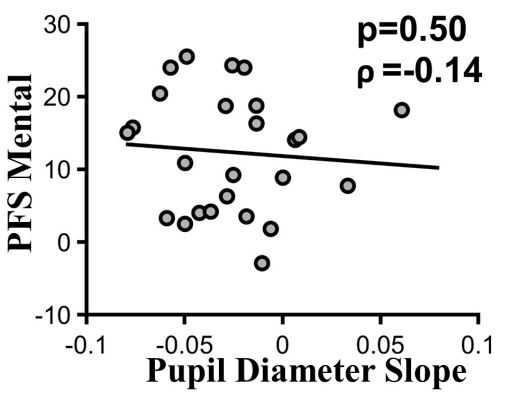

FIGURE 3 | Task-based fatigability. (A) Group mean subjective fatigue score by 20-min time blocks, $F_{(8,325)}=5.83, p<0.001$, effect size $>3$. (B) Linear fits to individual subjective fatigue scores as function of time-on-task. (C) Subjective fatigability [slopes of the linear fits in (B)] versus PFS mental subscores ( $\rho=-0.07$, $p=0.71$ ). (D) Group mean RT by 20-min time blocks, $F_{(7,169)}=3.71, p=0.001$, effect size $=0.24$. (E) Linear fits to individual reaction time as function of time-on-task. (F) Behavioral fatigability [slopes of the linear fits in (E)] versus PFS mental subscores $(\rho=-0.21, p=0.27)$. (G) Group mean pupil diameter by 20-min time blocks, $F_{(7,134)}=6.86, p<0.001$, effect size $=1.33$. (H) Linear fits to individual pupil diameter as function of time-on-task. (I) Autonomic fatigability [slopes of the linear fits in $\mathbf{( H )}$ ] versus PFS mental subscores $(\rho=-0.14, p=0.50)$.

the individual subject level, linear fit to RT measured in 20min blocks was calculated and shown in Figure 3E. Slopes of the linear fits were defined as behavioral fatigability. There was no correlation between behavioral fatigability and PFS mental fatigability subscore $(\rho=-0.21, p=0.27)$ (Figure 3F).

\section{Relation Between PFS Mental Fatigability Subscore and Task-Based Autonomic Fatigability}

As time-on-task progressed, pupil diameter decreased significantly across 20 -min time blocks, where $F_{(7,134)}=6.86$, $p<0.001$, and effect size $=1.33$ (Figure 3G). At individual subject level, linear fit to pupil diameters measured in 20-min blocks was calculated, and shown in Figure $3 \mathbf{H}$. Autonomic fatigability, defined as the slopes of the linear fits, did not correlate with PFS mental fatigability subscore $(\rho=-0.14$, $p=0.50)$ (Figure 3I).

\section{DISCUSSION}

We attempted to validate the PFS mental fatigability subsection using task-based fatigabilities. Prolonged performance of the cued Stroop task induced cognitive fatigue. As time-on-task progressed, subjective fatigue scores increased, reaction time slowed, and pupil diameter decreased. Accuracy, however, remained stable throughout the task. It is possible that a speedaccuracy tradeoff took place because it is known that older adults have the tendency to trade speed in favor of higher accuracy (Salthouse, 1979; Forstmann et al., 2011). Individual slopes of the linear fits to these measures as function of time-on-task were defined as task-based fatigabilities. The main finding of this work is that scores on the mental subsection of the PFS did not correlate with any of the three task-based fatigabilities. In contrast, PFS mental fatigability subscores were highly correlated with other instruments that measure cognitive fatigue (the FSS and the MFIS) and physical fatigue/fatigability (subsections of 
the PFS and of the MFIS) outside of the context of specifically defined activity amount and duration. The lack of correlation between general fatigue survey measures and task-based selfreports has been found before (Sandry et al., 2014). To our knowledge, however, this study is the first to test a questionnairebased measure of mental fatigability against task-based cognitive fatigabilities.

How to explain the lack of relation between PFS mental subscore and task-based fatigability? A potential issue in the PFS that may be hindering efficacy is that physical and cognitive fatigability are often confused for each other in common vernacular because research to date has not produced consistent nomenclature (Chaudhuri and Behan, 2004; Kluger et al., 2013). Therefore, participants may have encountered conflicting terms from professionals when referencing physical and cognitive domains. Specifically, in the PFS participants are asked to rate the imagined fatigue levels for a given activity without actually going through the activity. They are asked to rate physical fatigability and mental fatigability individually based on the one activity, which has both physical and mental dimensions. In our data, the physical and cognitive PFS subscores are highly correlated, suggesting that participants may be conflating the two when rating the physical and cognitive fatigability levels associated with the imagined example activity.

Another issue could be that proper assessment of cognitive fatigability requires a clear understanding of cognitive effort (Boksem and Tops, 2008) within a specific, quantifiable amount of activity (Eldadah, 2010). In the cognitive domain, perceived effort is influenced by motivation (van der Linden, 2011; Hopstaken et al., 2016) that is itself affected by the level of task enjoyment (Puca and Schmalt, 1999; Boksem and Tops, 2008). These factors are highly influential in perception of effort but have been hard to define in the cognitive domain; there is not yet a consensus in the literature as to the proper definition (van der Linden, 2011). In the PFS, the example activities are very specific and quantifiable with respect to physical effort for a task, such as sitting watching television for an hour. However, the amount of mental effort required for this activity may vary based on the type of program that is being watched or similar factors that impact how enjoyable it is to the individual. Therefore, the scale may benefit from using cognitive tasks that minimize the influence of personal preference. Since motivation is a very important factor in fatigue (Boksem et al., 2006; Gergelyfi et al., 2015; Hopstaken et al., 2016), it should be better qualified within questions that ask an individual to rate a task. For example, the questions on the PFS might clarify activities that a task is one that "you find enjoyable" or that "you consider to be a chore" and provide several examples within a similar range of intensity. With better characterization of the type of activity that is being asked about, the effect of motivation can be taken into account rather than varying between individuals based on interpretation. The authors of the PFS do a very good job of making these kinds of specifications for the more physically oriented activities, but the cognitively oriented activities are more multifaceted, and thus more difficult to specify.

The study's limitations include that the Stroop task does not impose time restrictions on responses within a trial; self-pace may reduce the reliability of objective measures (reaction time and pupil diameter). Also, behavioral fatigability measures have been tightly linked to confounding factors, such as motivation (Hopstaken et al., 2015) or compensation (Christodoulou, 2005; Wang et al., 2014), so may be more difficult to directly interpret than subjective fatigability. Finally, pupil data should be interpreted with caution as it reflects the collective contribution of numerous brain systems and structures (Mathôt, 2018).

\section{CONCLUSION}

The lack of correlation between task-based fatigability measures and the PFS mental subscore may indicate that mental fatigability, as a more multifaceted construct, is difficult to capture using questions asking about fatigue as a result of previous or imagined experiences. Therefore, more detailed and specific questions that take into account factors such as motivation and preference may be necessary to make this scale a viable clinical tool.

\section{ETHICS STATEMENT}

This study was carried out in accordance with the recommendations of the NIH Protection of Human Research Subjects and HIPPA for research guidelines with written informed consent from all subjects. All subjects gave written informed consent in accordance with the Declaration of Helsinki. The protocol was approved by the University of Florida Gainesville Health Science Center Institutional Review Board.

\section{AUTHOR CONTRIBUTIONS}

SB did conception, design, recruitment, data collection, data analysis, data interpretation, and manuscript preparation. IBHS did conception, design, data collection, data analysis, data interpretation, and manuscript preparation. QZ and JC did data analysis. RC did data interpretation and manuscript preparation. $\mathrm{BK}$ and $\mathrm{MD}$ did conception, design, data interpretation, and manuscript preparation.

\section{FUNDING}

This work was supported by NIH grant AG044862, the UF Institute on Aging, the McKnight Foundation, and the National Center for Advancing Translational Sciences of the National Institutes of Health under University of Florida Clinical and Translational Science Awards TL1TR001428 and UL1TR001427.

\section{ACKNOWLEDGMENTS}

We wish to thank Drs. Michael Marsiske and Todd Manini for helping with study design and participant recruitment. 


\section{REFERENCES}

Boksem, M. A. S., Meijman, T. F., and Lorist, M. M. (2006). Mental fatigue, motivation and action monitoring. Biol. Psychol. 72, 123-132. doi: 10.1016/j. biopsycho.2005.08.007

Boksem, M. A. S., and Tops, M. (2008). Mental fatigue: costs and benefits. Brain Res. Rev. 59, 125-139. doi: 10.1016/j.brainresrev.2008.07.001

Buysse, D. J., Reynolds, C. F., Monk, T. H., Berman, S. R., and Kupfer, D. J. (1989). The pittsburgh sleep quality index: a new instrument for psychiatric practice and research. Psychiatry Res. 28, 193-213. doi: 10.1016/0165-1781(89) 90047-4

Cathébras, P. J., Robbins, J. M., Kirmayer, L. J., and Hayton, B. C. (1992). Fatigue in primary care: prevalence, psychiatric comorbidity, illness behavior, and outcome. J. Gen. Intern. Med. 7, 276-286. doi: 10.1007/BF02598083

Chaudhuri, A., and Behan, P. O. (2004). Fatigue in neurological disorders. Lancet 363, 978-988. doi: 10.1016/S0140-6736(04)15794-2

Christodoulou, C. (2005). “The assessment and measurement of fatigue," in Fatigue as a Window to the Brain. ed. J. DeLuca (New York, NY: MIT Press), 19-35.

Eldadah, B. A. (2010). Fatigue and fatigability in older adults. PMぬR J. Inj. Funct. Rehabil. 2, 406-413. doi: 10.1016/j.pmrj.2010.03.022

Fisk, J. D., Ritvo, P. G., Ross, L., Haase, D. A., Marrie, T. J., and Schlech, W. F. (1994). Measuring the functional impact of fatigue: initial validation of the fatigue impact scale. Clin. Infect. Dis. 18(Suppl. 1), S79-S83. doi: 10.1093/ clinids/18.Supplement_1.S79

Forstmann, B. U., Tittgemeyer, M., Wagenmakers, E.-J., Derrfuss, J., Imperati, D., and Brown, S. (2011). The speed-accuracy tradeoff in the elderly brain: a structural model-based approach. J. Neurosci. 31, 17242-17249. doi: 10.1523/ JNEUROSCI.0309-11.2011

Gergelyfi, M., Jacob, B., Olivier, E., and Zénon, A. (2015). Dissociation between mental fatigue and motivational state during prolonged mental activity. Front. Behav. Neurosci. 9:176. doi: 10.3389/fnbeh.2015.00176

Gill, T. M., Desai, M. M., Gahbauer, E. A., Holford, T. R., and Williams, C. S. (2001). Restricted activity among community-living older persons: incidence, precipitants, and Health Care Utilization. Ann. Intern. Med. 135, 313-321. doi: 10.7326/0003-4819-135-5-200109040-00007

Glynn, N. W., Santanasto, A. J., Simonsick, E. M., Boudreau, R. M., Beach, S. R., Schulz, R., et al. (2015). The pittsburgh fatigability scale for older adults: development and validation. J. Am. Geriatr. Soc. 63, 130-135. doi: 10.1111/jgs. 13191

Hardy, S. E., and Studenski, S. A. (2008a). Fatigue and function over 3 years among older adults. J. Gerontol. A Biol. Sci. Med. Sci. 63, 1389-1392. doi: 10.1093/ gerona/63.12.1389

Hardy, S. E., and Studenski, S. A. (2008b). Fatigue predicts mortality in older adults. J. Am. Geriatr. Soc. 56, 1910-1914. doi: 10.1111/j.1532-5415.2008.01957.x

Holm, S. (1979). A simple sequentially rejective multiple test procedure. Scand. J. Stat. 6, 65-70.

Hopstaken, J. F., Van der Linden, D., Bakker, A. B., and Kompier, M. A. J. (2015). A multifaceted investigation of the link between mental fatigue and task disengagement. Psychophysiology 52, 305-315. doi: 10.1111/psyp.12339

Hopstaken, J. F., van der Linden, D., Bakker, A. B., Kompier, M. A. J., and Leung, Y. K. (2016). Shifts in attention during mental fatigue: evidence from subjective, behavioral, physiological, and eye-tracking data. J. Exp. Psychol. Hum. Percept. Perform. 42, 878-889. doi: 10.1037/xhp0000189

Jensen, A. R., and Rohwer, W. D. (1966). The stroop color-word test: a review. Acta Psychol. 25, 36-93. doi: 10.1016/0001-6918(66)90004-7

Johns, M. W. (1991). A new method for measuring daytime sleepiness: the Epworth sleepiness scale. Sleep 14, 540-545. doi: 10.1016/j.sleep.2007.08.004
Kluger, B. M., Krupp, L. B., and Enoka, R. M. (2013). Fatigue and fatigability in neurologic illnesses: proposal for a unified taxonomy. Neurology 80, 409-416. doi: 10.1212/WNL.0b013e31827f07be

Krupp, L. B., LaRocca, N. G., Muir-Nash, J., and Steinberg, A. D. (1989). The fatigue severity scale: application to patients with multiple sclerosis and systemic lupus erythematosus. Arch. Neurol. 46, 1121-1123. doi: 10.1001/archneur.1989. 00520460115022

Mathôt, S. (2018). Pupillometry: psychology, physiology, and function. J. Cogn. 1:16 doi: $10.5334 /$ joc. 18

Muscio, B. (1921). Is a fatigue test possible? Br. J. Psychol. Gen. Sect. 12, 31-46. doi: 10.1111/j.2044-8295.1921.tb00036.x

Nasreddine, Z. S., Phillips, N. A., Bédirian, V., Charbonneau, S., Whitehead, V., Collin, I., et al. (2005). The montreal cognitive assessment, MOCA: a brief screening tool for mild cognitive impairment. J. Am. Geriatr. Soc. 53, 695-699. doi: 10.1111/j.1532-5415.2005.53221.x

Puca, R. M., and Schmalt, H.-D. (1999). Task enjoyment: a mediator between achievement motives and performance. Mot. Emot. 23, 15-29. doi: 10.1023/A: 1021327300925

Salthouse, T. A. (1979). Adult age and the speed-accuracy trade-off. Ergonomics 22, 811-821. doi: 10.1080/00140137908924659

Sandry, J., Genova, H. M., Dobryakova, E., DeLuca, J., and Wylie, G. (2014). Subjective cognitive fatigue in multiple sclerosis depends on task length. Front. Neurol. 5:214. doi: 10.3389/fneur.2014.00214

Unsworth, N., Heitz, R. P., Schrock, J. C., and Engle, R. W. (2005). An automated version of the operation span task. Behav. Res. Methods 37, 498-505. doi: 10 . 3758/BF03192720

van der Linden, D. (2011). "The urge to stop: the cognitive and biological nature of acute mental fatigue," in Cognitive Fatigue: Multidisciplinary Perspectives on Current Research and Future Applications, ed. P. L. Ackerman (Washington, DC: American Psychological Association), 149-164. doi: 10.1037/123 43-007

van der Linden, D., Frese, M., and Meijman, T. F. (2003). Mental fatigue and the control of cognitive processes: effects on perseveration and planning. Acta Psychol. 113, 45-65. doi: 10.1016/S0001-6918(02)00150-6

Wang, C., Ding, M., and Kluger, B. M. (2014). Change in intraindividual variability over time as a key metric for defining performance-based cognitive fatigability. Brain Cogn. 85, 251-258. doi: 10.1016/j.bandc.2014.01.004

Ware, J. E. Jr., and Sherbourne, C. D. (1992). The MOS 36-item short-form health survey (SF-36): I. conceptual framework and item selection. Med. Care 30, 473-483.

Zigmond, A. S., and Snaith, R. P. (1983). The hospital anxiety and depression scale. Acta Psychiatr. Scand. 67, 361-370. doi: 10.1111/j.1600-0447.1983.tb09716.x

Conflict of Interest Statement: The authors declare that the research was conducted in the absence of any commercial or financial relationships that could be construed as a potential conflict of interest.

The handling Editor declared a shared affiliation, though no other collaboration, with several of the authors SB, IBHS, QZ, JC, RC, and MD at the time of review.

Copyright (c) 2018 Burke, Babu Henry Samuel, Zhao, Cagle, Cohen, Kluger and Ding. This is an open-access article distributed under the terms of the Creative Commons Attribution License (CC BY). The use, distribution or reproduction in other forums is permitted, provided the original author(s) and the copyright owner(s) are credited and that the original publication in this journal is cited, in accordance with accepted academic practice. No use, distribution or reproduction is permitted which does not comply with these terms. 